\title{
Observation of bipolar moving magnetic features streaming out from a naked spot
}

\author{
F. Zuccarello ${ }^{1}$, P. Romano ${ }^{2}$, S. L. Guglielmino ${ }^{1}$, M. Centrone ${ }^{3}$, S. Criscuoli ${ }^{3}$, I. Ermolli ${ }^{3}$, F. Berrilli ${ }^{4}$, and D. Del Moro ${ }^{4}$ \\ 1 Dipartimento di Fisica e Astronomia - Sezione Astrofisica, Università di Catania, via S. Sofia 78, 95123 Catania, Italy \\ e-mail: fzu@oact. inaf.it \\ 2 INAF - Osservatorio Astrofisico di Catania, via S. Sofia 78, 95123 Catania, Italy \\ 3 INAF - Osservatorio Astronomico di Roma, via Frascati 33, 00040, Monte Porzio Catone, Italy \\ 4 Department of Physics, University of Roma Tor Vergata, via della Ricerca Scientifica 1, 00133 Roma, Italy
}

Received 4 April 2009 / Accepted 28 April 2009

ABSTRACT

\begin{abstract}
Context. Mechanisms responsible for active-region formation, evolution, and decay have been investigated by many authors and several common features have been identified. In particular, a key element in the dispersal of the magnetic field seems to be the presence of magnetic elements, called moving magnetic features (MMFs).

Aims. We analyze the short-lived sunspot group NOAA 10977, which appeared on the solar disk between 2 and 8 December 2007, to study the details of its emergence and decay phases.

Methods. We performed a multi wavelength analysis of the region using images at visible ( $\mathrm{G}$ band and $\mathrm{H}_{\alpha}$ ) and near-IR (Ca II) wavelengths acquired by both the IBIS instrument and SOT/HINODE, EUV images $(17.1 \mathrm{~nm})$ acquired by TRACE, and MDI and SOT magnetograms.

Results. The observed region exhibits some peculiarities. During the emergence phase the formation of the f-pore was initially observed, while the p-polarity later formed a naked spot, i.e., a sunspot without a penumbra. We measured a moat flow around this spot, and observed some MMFs streaming out from it during the decay phase. The characteristics of these MMFs allowed us to classify them as type I (U-shaped) MMFs. They were also cospatial with sites of increased brightness both in the photosphere and the chromosphere.

Conclusions. The presence of bipolar MMFs in a naked spot indicates that current interpretation of bipolar MMFs, as extensions of the penumbral filaments beyond the sunspot outer boundaries, should be revised, to take into account this observational evidence. We believe that our results provide new insights into improving models of sunspot evolution.
\end{abstract}

Key words. Sun: activity - Sun: sunspots - Sun: magnetic fields

\section{Introduction}

The study of the physical processes responsible for the formation, evolution, and decay of solar active regions (ARs) provides important information about the modalities of interaction between high ionized plasma and magnetic fields. In spite of the large number of studies of these processes, carried out by both data analyses (van Driel-Gesztelyi 2002; Zuccarello et al. 2009) and numerical simulations (Cheung et al. 2008, and references therein), several aspects remain unclear. For instance, it remains unclear the role played by the so-called moving magnetic features (MMFs, hereafter), small magnetic-flux concentrations, which are usually observed streaming out from the sunspot penumbra, later interacting with the ambient magnetic field, and then disappearing (Sheeley 1969; Harvey \& Harvey 1973).

MMFs have been classified into the following types. Type I are bipolar structures, of typical velocities $\sim 0.5-1 \mathrm{~km} \mathrm{~s}^{-1}$, which can be further divided into two classes: $\Omega$-shaped, which has an inner footpoint of the same polarity as the sunspot, and Ushaped, which has an inner footpoint of opposite polarity to the sunspot. Type II MMFs are single magnetic elements with the same polarity as the sunspot in which they originate, and velocities of $\sim 0.5-1 \mathrm{~km} \mathrm{~s}^{-1}$. Finally, type III MMFs are single magnetic elements of opposite polarity with respect to the relevant sunspot, and velocities in the range $\sim 2-3 \mathrm{~km} \mathrm{~s}^{-1}$ (Shine \& Title 2001). Bernasconi et al. (2002) also observed MMFs flowing toward the sunspot.

Based on high-resolution observations, analyses of MMF characteristics have demonstrated that some MMFs are manifestations of the penumbral Evershed flow in the sunspot moat, supporting earlier results about the magnetic connection between MMFs and the sunspot penumbra (see, e.g., Yurchyshyn et al. 2001; Zhang et al. 2003; Sainz Dalda \& Martínez Pillet 2005; Cabrera Solana et al. 2006; Kubo et al. 2007). On the other hand, statistical analyses of sunspot groups also showed type II MMFs flowing out from naked spots, i.e., sunspots without a penumbra (Harvey \& Harvey 1973). Independently of both the presence of penumbra and of the evolutionary stage, Brickhouse \& LaBonte (1988) also showed that sunspots can be surrounded by MMFs, and that neither the number of observed features, nor the direction of their motion, correlates with the sunspot or moat properties. We note that Brickhouse \& LaBonte (1988) did not specify whether the analyzed MMFs were single magnetic elements or bipolar structures.

We report the detection of bipolar MMFs streaming out from a naked spot. We present the observations analyzed in Sect. 2. 
The results obtained are presented and discussed in Sect. 3. Section 4 summarizes our conclusions.

\section{Observations and data reduction}

Observations analyzed in this study were collected during a coordinated campaign of HINODE/SOT and DST/IBIS between 2 and 8 December 2007. Auxiliary observations taken over the same period with SOHO/MDI and TRACE were also analyzed.

HINODE - The active region NOAA 10977 was observed by the Solar Optical Telescope (SOT; Tsuneta et al. 2008) onboard the Hinode satellite (Kosugi et al. 2007), during the Hinode Operation Plan 35, which occurred between 5 and 7 December 2007. We discuss here results obtained by the analysis of observations taken on 5 December 2007. The SOT Broad-band Filter Imager (BFI) acquired filtergrams in the core of the Ca II $\mathrm{H}$ line $(396.85 \pm 0.3 \mathrm{~nm})$ and in the $\mathrm{G}$ band $(430.5 \pm 0.8 \mathrm{~nm})$, with a FoV of $\sim 110^{\prime \prime} \times 110^{\prime \prime}$, and a spatial sampling of $0.1^{\prime \prime} /$ pixel. The Narrow-band Filter Imager (NFI) obtained Stokes $I$ and $V$ filtergrams in the blue wing of the Na I D1 line $(589.6 \mathrm{~nm})$, with a spatial sampling of $0.16^{\prime \prime} /$ pixel and covering the same FoV of BFI filtergrams. The cadence of both the NFI images and the cotemporal G-band and Ca II H filtergrams was two minutes between both 10:43 and 11:17 UT, and 14:06 and 15:48 UT. SOT images were corrected for dark current, flat field, and cosmic rays with the standard IDL SolarSoft routines and coaligned following Shimizu et al. (2007). Magnetograms were derived from the NaI D1 Stokes $I$ and $V$ images acquired in the blue wing of the line, by making use of the weak-field approximation (Stix 2002) described by Guglielmino et al. (2008). By calculating the noise level in quiet Sun regions, we also estimated the error per pixel in the flux measurement to be $\sim 3 \mathrm{Mx} \mathrm{cm}^{-2}$. Finally, we have applied the LCT method (Yi \& Molowny-Horas 1995) with a $F W H M$ window of $0.3^{\prime \prime} \times 0.3^{\prime \prime}$ and a time interval of two minutes, to G-band images acquired between 14:06 and 15:48 UT to determine the horizontal velocity fields in the region analyzed.

$I B I S$ - We analyzed data acquired between 3 and 5 December 2007 with the Interferometric Bidimensional Spectrometer (IBIS Cavallini et al. 2006), installed at the Dunn Solar Telescope of the US National Solar Observatory. The dataset consists of 320 sequences along the profiles of the Fe I line ( $\lambda=709.00 \mathrm{~nm}, 29$ spectral points $)$ and the Ca II line $(\lambda=$ $854.21 \mathrm{~nm}, 39$ spectral points), acquired between 16:40 and 18:17 on 3 December 2007, 15:36 and 17:19 on 4 December 2007 and between 15:11 and 17:10 on 5 December 2007. The pixel scale was $0.17^{\prime \prime}$, and the exposure time $20 \mathrm{~ms}$. The time interval between two successive spectral series was $\sim 20 \mathrm{~s}$. Data reduction, which includes standard CCD response calibration and correction for systematic blueshift of the instrument profile, was performed as described by Giordano et al. (2008).

MDI - MDI (Scherrer et al. 1995) magnetograms, characterized by a spatial resolution of $\sim 1.96^{\prime \prime}$ and a time cadence of 96 min, were taken between 2 and 9 December 2007. We selected a box centered on the analyzed region and corrected all the magnetograms for the angle between the magnetic-field direction and the observer line-of-sight, and for the underestimation of the flux density following the results of Berger \& Lites (2003). We aligned all the sub-fields of the magnetograms by applying a standard differential rotation rate (Howard et al. 1990) with a sampling of 1 arcsec. We calculated the positive and negative magnetic fluxes inside the box $\Phi_{+(-)}=\int B_{z} \mathrm{~d} S$, where $B_{z}$ is the intensity of the magnetic field along the line-of-sight, and $S$ is the area of the box. Figure 1 shows the temporal evolution in

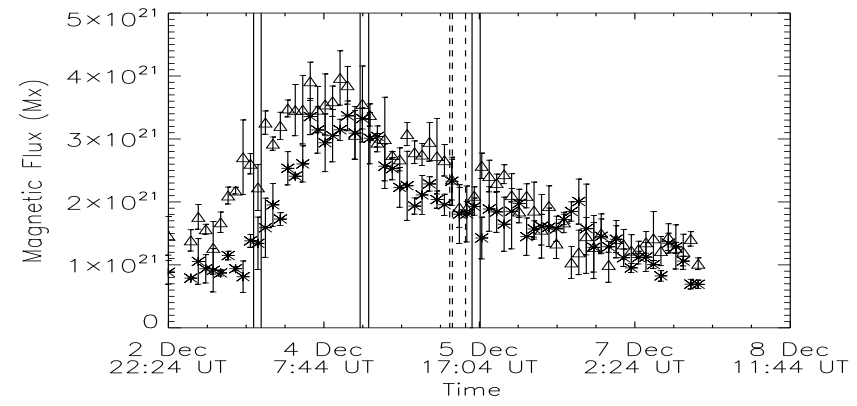

Fig. 1. Magnetic flux in NOAA 10977 during the active-region lifetime. Triangles (crosses) indicate the positive (negative) magnetic flux. Bars indicate the errors in the measure of the magnetic flux. Vertical continuous lines report the time intervals covered by IBIS observations, while dotted lines indicate the time intervals covered by SOT/NFI in the $586.9 \mathrm{~nm}$ line.

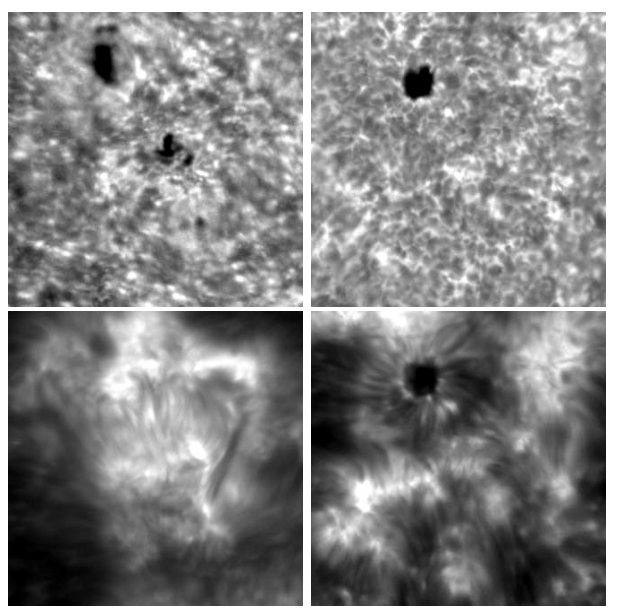

Fig. 2. Images obtained with the filtergrams acquired with IBIS in the Fe I line (top) and in the Ca II line (bottom). The images of 3 December (on the left) show both following and preceding polarities, while the images of 5 December (on the right) show only the preceding naked spot. The field of view is $\sim 40 \times 40 \mathrm{Mm}^{2}$. In this and the following images, North is at the top, West at the right.

the positive (triangles) and negative (crosses) magnetic flux of NOAA 10977.

TRACE - TRACE (Handy et al. 1999) $17.1 \mathrm{~nm}$ images of the region were taken on 5 December in full-resolution mode, $768 \times 768$ pixels, with a pixel size of $0.5^{\prime \prime}$ and a FoV of $6.4^{\prime} \times$ $6.4^{\prime}$; the time cadence was $\sim 30 \mathrm{~s}$. Data were corrected for instrumental effects, such as pedestal and dark current, and for readout noise using standard IDL SolarSoft routines.

\section{Results}

The active region NOAA 10977 appeared on the solar disk on 2 December 2007 and disappeared on 8 December. It exhibited a simple bipolar configuration and no flare activity. The magnetic flux (Fig. 1) increased rapidly during the first two days (emergence phase) and successively decreased with a smoother trend (decay phase).

Emergence phase - The f-pore was the first to appear. On 3 December, it exhibited a north-south elongated shape in the Fe I line image acquired by IBIS (Fig. 2, top-left). The p-spot appears initially at the south-west of the f-pore in the form of a couple of very small pores. In this phase, an Arch Filament System (AFS, hereafter) is observed between the f-pore and the 


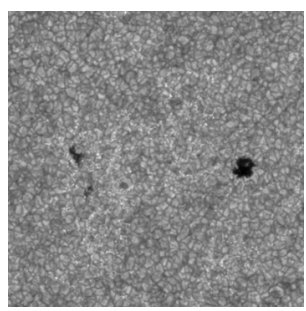

(a)

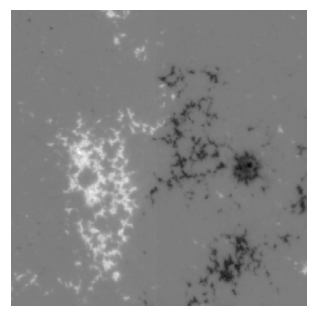

(b)

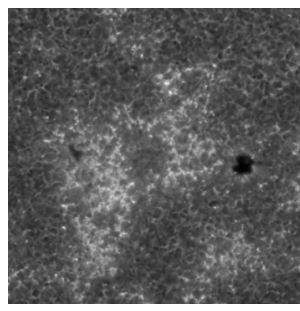

(c)

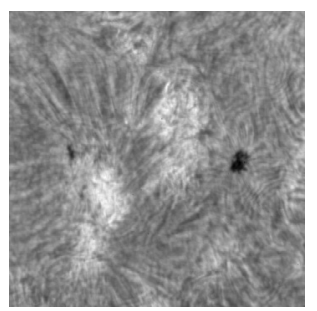

(d)

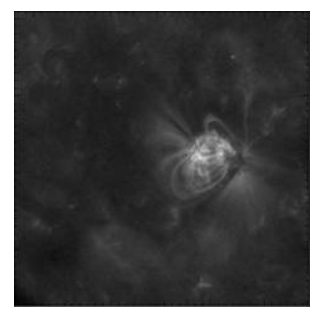

(e)

Fig. 3. Configuration of the active region at several atmospheric layers on 5 December 2007. From left to right: a) Hinode image in the G band (15:30 UT); b) Hinode Na I D1 magnetogram (15:30 UT); c) Hinode image in the Ca II H line (15:30 UT); d) Hinode image in the $\mathrm{H}_{\alpha}$ line (16:30 UT). The field of view of these images is $\sim 60 \times 60 \mathrm{Mm}^{2}$. e) TRACE $17.1 \mathrm{~nm}$ image (19:13 UT). The FoV is $384^{\prime \prime} \times 384^{\prime \prime}$ and the spatial sampling is $0.5^{\prime \prime} /$ pixel.

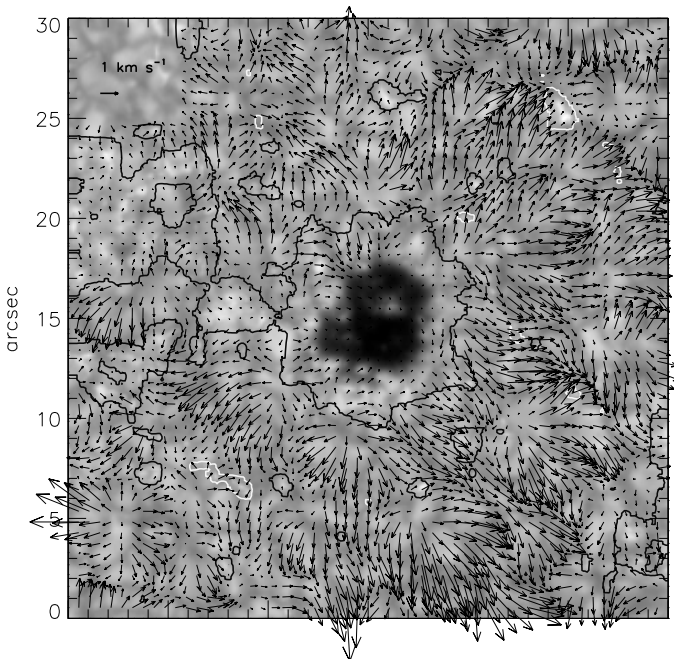

Fig. 4. Horizontal velocity fields (60 min average), derived by the LCT technique, overplotted on the Hinode G-band image acquired on 5 December 2007 at 15:40 UT. The arrows indicate the direction and the intensity of the horizontal velocity in the surroundings of the preceding naked spot. The arrow in the left-top corner represents a velocity of $1 \mathrm{~km} \mathrm{~s}^{-1}$. The black (white) isocontours indicate the negative (positive) contours of the magnetic field $\left[25(-25) \mathrm{Mx} \mathrm{cm}^{-2}\right]$. The presence of ordered diverging motions from the naked spot is evident.

preceding small pores. This AFS is oriented north-south and not in the usual east-west direction (see Fig. 2 bottom-left). In subsequent days, the f-pore and the p-spot were however east-west aligned, indicating that at the end of the emerging process the flux tubes are directed, as usually, in a direction almost parallel to the solar equator. This behavior was confirmed by TRACE images (see Fig. 3e) and MDI maps.

Decay phase - During 4-8 December 2007, the AR was in its decay phase (Fig. 1). In Fig. 3, we can see the configuration of the active region (AR) at several atmospheric levels on 5 December 2007. In particular, the SOT G-band image (Fig. 3a) indicates that the following (positive) polarity is a pore, while the preceding (negative) polarity is constituted by a small naked spot (diameter $\sim 4000 \mathrm{~km}$ ). This region was without penumbra throughout its lifetime. The SOT Na I D1 magnetogram (Fig. 3b) shows the magnetic configuration of the AR: we can see that the negative magnetic-field concentration, corresponding to the naked spot, shows several relatively small filamentary extrusions that however do not correspond to any penumbral structure in the G-band image. The Ca II H image (Fig. 3c) shows a bright pattern distributed on both the positive and negative diffuse fields, in addition to a bright ring encircling the naked spot. The bright (facular) pattern is also visible in the $\mathrm{H}_{\alpha}$ image (Fig. 3d). The TRACE $17.1 \mathrm{~nm}$ image (Fig. 3e) shows bright coronal loops connecting the f-pore and the naked spot.

Figure 4 shows the map of the horizontal velocity fields, derived by applying the LCT technique to Hinode G-band images, in the surrounding of the preceding naked spot. The presence of ordered large-scale outflows from a region approximately encircling the naked spot suggests that moat flows are present even in the absence of a penumbra, in disagreement with results presented in the literature (e.g., Vargas Domínguez et al. 2008).

The analysis of SOT magnetograms also indicates the presence of four bipolar MMFs around the preceding naked spot (see Fig. 5a). More precisely, a first MMF (named $A$ ) was observed at 15:00 UT, whcih appeared to be expelled from the eastsouthern part of the naked spot and then move away following an arched C-shaped path. After $20 \mathrm{~min}$, two more MMFs ( $B$ and $C$ ) were seen to stream out from the west-northern part of the naked spot, and after $\sim 20$ min a fourth MMF $(D)$ was observed on the west side of the naked spot. The MMFs appear unevenly around the spot, in agreement with both previous observations (e.g., Ryutova \& Hagenaar 2007; Ryutova et al. 2007) and model results from numerical simulations showing that magnetic solitons may appear anywhere (Ryutova et al. 1997). We note that the successive development of these and possibly other MMFs could not be investigated because of the end of the observing run.

All of these MMFs exhibit a polarity opposite to the main magnetic-flux bundle initially closer to the naked spot and are therefore classified as U-shaped type I MMF. Analysis of SOT G-band images show bright knots spatially coincident with MMFs $A$ and $B$ (see Fig. 5b), while in the Ca II H line images, all the MMFs are cospatial with brightness enhancements (see Fig. 5c).

Inspection of Fig. 4 reveals that the MMFs are located in sites of surface divergence flows, which carry them through the boundaries of the moat. We note the arched C-shaped flow related to the site of the MMF A, which followed an analogous path in the magnetogram sequence.

Velocities of the MMFs were also estimated by linearly fitting time and distance (measured from the border of the naked spot) data. We found typical velocity values of $0.7 \mathrm{~km} \mathrm{~s}^{-1}$, in agreement with values found by the LCT $\left(0.6-1.0 \mathrm{~km} \mathrm{~s}^{-1}\right)$ technique. We found that only MMF $\mathrm{B}$ decelerates during the observation as it moves further. This finding, with the observed chromospheric brightening, would support the soliton model by Ryutova \& Hagenaar (2007). Following this model, we obtained a dissipation time of $\sim 17 \mathrm{~min}$ by fitting the contrast in $\mathrm{Ca}$ II $\mathrm{H}$ line for MMF A during its decay. 

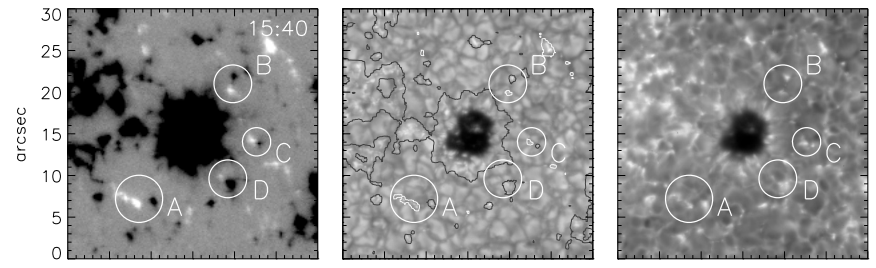

Fig. 5. From left to right: a) Hinode magnetogram showing the MMFs flowing out of the negative polarity associated with the preceding naked spot. The circles indicate the locations of MMFs $A, B, C$, and $D$. b) Hinode G-band image showing the position of the MMFs; MMFs $A$ and $B$ coincide with sites of brightness increase; black (white) contours indicate negative (positive) polarities. c) Hinode $\mathrm{Ca}$ II $\mathrm{H}$ line image, showing that at this wavelength all the observed MMFs are associated with an increased emission. The FoV is the same as Fig. 4.

\section{Discussion and conclusions}

We have found that NOAA 10977 exhibits some peculiarities compared to most of the regions analyzed so far. For instance, during the emerging phase the first polarity that gives rise to major pores is the following one. An AFS is observed initially aligned in the north-south direction (instead of the usual eastwest direction), while in the following days the AFS is correctly oriented.

During the decay phase, we found moat flows and U-shaped bipolar MMFs flowing out of the preceding naked spot. Bright knots at photospheric and chromospheric levels were observed in sites cospatial with the MMFs, confirming previous observations reported by Harvey \& Harvey (1973) and Shine \& Title (2001).

Concerning the occurrence of the bipolar MMFs around sunspots, we recall that Harvey \& Harvey (1973) suggested that, at the sunspot outer edge, the sub-photospheric field lines can be irregularly twisted due to the interaction with the (super)granular velocity field. When the outer and twisted flux bundle becomes separated from the main sunspot field, it is brought upward from the (super)granular velocity field and the twisted parts may appear in the photosphere as MMFs. Weiss et al. (2004) interpreted type I MMFs as the footpoints of magnetic loops belonging to the sunspot penumbra that erupt in the photosphere along a submerged (downward-pumped) flux tube in sites characterized by strong convective updrafts. Successively, these loops are swept outward by the moat flow, along with the granulation pattern. More generally, the interpretations of type I MMFs usually found in the literature refer to the presence of the penumbral filaments, which in our high-resolution observations were however never detected. The study carried out by Vargas Domínguez et al. (2008) also found evidence of penumbra in moat flow regions.

We conclude that observations of both moat flows and bipolar MMFs in a naked spot, provides useful information for improving models of sunspot evolution.

Acknowledgements. This work was supported by the Istituto Nazionale di Astrofisica (INAF), by the Catania University, by the Agenzia Spaziale Italiana (contract I/015/07/0) and by the European Commission through the SOLAIRE Network (MRTN-CT-2006-035484).

\section{References}

Berger, T. E., \& Lites, B. W. 2003, Sol. Phys., 213, 213

Bernasconi, P. N., Rust, D. M., Georgoulis, M. K., \& LaBonte, B. J. 2002, Sol. Phys., 209, 119

Brickhouse, N. S., \& LaBonte, B. J. 1988, Sol. Phys., 115, 43

Cabrera Solana, D., Bellot Rubio, L. R., Beck, C., \& del Toro Iniesta, J. C. 2006, ApJ, 649, L41

Cavallini, F. 2006, Sol. Phys., 236, 415

Cheung, M. C. M., Schüssler, M., Tarbell, T. D., \& Title, A. M. 2008, ApJ, 687, 1373

Giordano, S., Berrilli, F., Del Moro, D., \& Penza, V. 2008, A\&A, 489, 747

Guglielmino, S. L., Zuccarello, F., Romano, P., \& Bellot Rubio, L. R. 2008, ApJ, 688, L111

Handy, B. N., Acton, L. W., Kankelborg, C. C., et al. 1999, Sol. Phys., 187, 229 Harvey, K. L., \& Harvey, J. 1973, Sol. Phys., 28, 61

Howard, R. F., Harvey, J. W., \& Forgach, S. 1990, Sol. Phys., 130, 295

Kosugi, T., Matsuzaki, K., Sakao, T., et al. 2007, Sol. Phys., 243, 3

Kubo, M., Ichimoto, K., Shimitzu, T., Tsuneta, S., et al. 2007, PASJ 59, S607

Ryutova, M. P., \& Hagenaar, H. 2007, Sol. Phys., 243, 281

Ryutova, M. P., Shine, R., Title, A., \& Sakai, J. I. 1997, ApJ, 492, 402

Ryutova, M. P., Hagenaar, H., \& Title, A. 2007, ApJ, 656, L45

Sainz Dalda, A., \& Martínez Pillet, V. 2005, ApJ, 632, 1176

Scherrer, P. H., Bogart, R. S., Bush, R. I., et al., Sol. Phys.162, 129

Sheeley, N. R. 1969, Sol. Phys., 9, 347

Shimizu, T., Katsukawa, Y., Matsuzaki, K., et al. 2007, PASJ, 59, 845

Shine, T., \& Title, A. 2001, in Encyclopedia of Astronomy and Astrophysics, ed.

P. Murdin (London: Nature Publishing; and Bristol: IOP Publishing), 4, 3209

Stix, M. 2002, The sun: an introduction - 2nd ed. (Berlin Springer)

Tsuneta, S., Ichimoto, K., Katsukawa, Y., et al. 2008, Sol. Phys., 249, 167

Vargas Domínguez, S., Rouppe van der Voort, L., Bonet, J. A., et al. 2008, ApJ, 679,900

van Driel-Gesztelyi, L. 2002, in SOLMAG 2002. Proc. of the Magnetic Coupling of the Solar Atmosphere Euroconf., IAU Colloq. 188, ESA SP-505, 113

Weiss, N. O., Thomas, J. H., Brummell, N. H., \& Tobias, S. M. 2004, ApJ, 600, 1073

Yi, Z., \& Molowny-Horas, R. 1995, A\&A, 295, 199

Yurchyshyn, V. B., Wang, H., \& Goode, P. R. 2001, ApJ, 550, 470

Zhang, H., Solanki, S. K., \& Wang, H. 2003, A\&A, 399, 755

Zuccarello, F., Guglielmino, S. L., Battiato, V., et al. 2009, Acta Geophys., 57, 15 